\title{
Resolution dependence on phase extraction by the Hilbert transform in phase calibrated and dispersion compensated ultrahigh resolution spectrometer-based OCT
}

\author{
Israelsen, Niels Møller; Maria, Michael; Feuchter, Thomas; Bradu, A.; Podoleanu, Adrian; Bang, Ole
}

Published in:

Proceedings of SPIE

Link to article, DOI:

$10.1117 / 12.2282295$

Publication date:

2018

Document Version

Publisher's PDF, also known as Version of record

Link back to DTU Orbit

Citation (APA):

Israelsen, N. M., Maria, M., Feuchter, T., Bradu, A., Podoleanu, A., \& Bang, O. (2018). Resolution dependence on phase extraction by the Hilbert transform in phase calibrated and dispersion compensated ultrahigh resolution spectrometer-based OCT. In Proceedings of SPIE (Vol. 10591). [105910D] SPIE - International Society for Optical Engineering. Proceedings of SPIE - The International Society for Optical Engineering https://doi.org/10.1117/12.2282295

\section{General rights}

Copyright and moral rights for the publications made accessible in the public portal are retained by the authors and/or other copyright owners and it is a condition of accessing publications that users recognise and abide by the legal requirements associated with these rights.

- Users may download and print one copy of any publication from the public portal for the purpose of private study or research.

- You may not further distribute the material or use it for any profit-making activity or commercial gain

- You may freely distribute the URL identifying the publication in the public portal 


\section{Resolution dependence on phase extraction by the Hilbert transform in phase calibrated and dispersion compensated ultrahigh resolution spectrometer-based OCT}

N. M. Israelsen, M. Maria, T. Feuchter, A. Bradu, A. Podoleanu, et al.

N. M. Israelsen, M. Maria, T. Feuchter, A. Bradu, A. Podoleanu, O. Bang, "Resolution dependence on phase extraction by the Hilbert transform in phase calibrated and dispersion compensated ultrahigh resolution spectrometer-based OCT," Proc. SPIE 10591, 2nd Canterbury Conference on OCT with Emphasis on Broadband Optical Sources, 105910D (5 March 2018); doi: 10.1117/12.2282295

Event: Second Canterbury Conference on Optical Coherence Tomography, 2017, Canterbury, United Kingdom 


\title{
Resolution dependence on phase extraction by the Hilbert transform in phase calibrated and dispersion compensated ultrahigh resolution spectrometer based OCT
}

\author{
N. M. Israelsen ${ }^{1 *}$, M. Maria ${ }^{2,3}$, T. Feuchter ${ }^{2}$, A. Bradu ${ }^{3}$, A. Podoleanu ${ }^{3}$ and O. Bang ${ }^{1}$ \\ ${ }^{1}$ DTU Fotonik, Department of Photonic s Engineering, Technical University of Denmark, \\ DK-2800 Kgs. Lyngby, Denmark \\ ${ }^{2}$ NKT Photonics A/S, Blokken 84, 3460 Birkerød, Denmark \\ ${ }^{3}$ Applied Optics Group, University of Kent, Canterbury CT2 7NH, UK \\ *corresponding author electronic address: nikr@fotonik.dtu.dk
}

\begin{abstract}
Ultrahigh resolution optical coherence tomography (UHR-OCT) is enabled by using a broad band source. Simultaneously, this makes the OCT image more sensitive to dispersion mismatch in the interferometer. In spectral domain OCT, dispersion left uncompensated in the interferometer and detector non-linearities lead together to an unknown chirp of the detected interferogram. One method to compensate for the chirp is to perform a pixel-wavenumber calibration versus phase that requires numerical extraction of the phase. Typically a Hilbert transform algorithm is employed to extract the optical phase versus wavenumber for calibration and dispersion compensation. In this work we demonstrate UHR-OCT at $1300 \mathrm{~nm}$ using a Super continuum source and highlight the resolution constraints in using the Hilbert transform algorithm when extracting the optical phase for calibration and dispersion compensation. We demonstrate that the constraints cannot be explained purely by the numerical errors in the data processing module utilizing the Hilbert transform but must be dictated by broadening mechanisms originating from the experimentally obtained interferograms.
\end{abstract}

Keywords: ultrahigh resolution OCT, dispersion compensation, spectrometer calibration

\section{INTRODUCTION}

By its 25 years anniversary, optical coherence tomography (OCT) has proven as a versatile technique spanning from ophthalmology and dermatology to non-destructive testing (NDT) [1,2]. Especially, spectral domain (SD)version of OCT has accelerated its applicability, contributing to major increases in sensitivity and imaging speed [2]. Essential for sensitivity and axial resolution, is a correct pixel-to-wavenumber calibration. The larger the bandwidth (as required by UHR-OCT), the larger the impact of chirp on both sensitivity and axial resolution. In order to approach the theoretical resolution limit, near-perfect compensation of dispersion mismatch and detector non-linearities are necessary.

A method for both calibration and dispersion compensation (DC) employs the linear relationship between the phase and the wavenumber $k=\frac{\omega}{c}$ where $\omega$ and $c$ are the optical frequency and the speed of light, respectively [3]. The wavenumber-phase relation can be written as

$$
\begin{aligned}
& \phi(i)=2 k_{r e l}(i) z+\phi_{0}(i), \text { where } \\
& k(i)=k_{a b s, i=n}+\left(k_{r e l}-k_{r e l, i=n}\right),
\end{aligned}
$$


$\phi$ is the total phase, $i$ is the pixel number on the camera of the spectrometer interferometer, $2 z$ is the optical path difference in the interferometer (measured in air), and $\phi_{0}$ is the phase distortion due to system dispersion and detector non-linearities. $k_{r e l}$ is the relative wavenumber ranging from zero to a finite value and $k_{a b s, i=n}$ is the absolute $k$-value of pixel $n$ calibrated by using a narrow band filter or a laser line. By recording two interferograms for two $z$-values, $z_{1}$ and $z_{2}$, a pixel to wavenumber relation can be obtained as:

$$
k_{\text {rel }}(i)=\frac{\phi_{2}(i)-\phi_{1}(i)}{2\left(z_{2}-z_{1}\right)}
$$

and eq. (2) if the phases $\phi_{1}$ and $\phi_{2}$ can be extracted from the interferograms. Subsequently, $\phi_{0}$ can be determined by exploiting eq. (1). In order to extract both $\phi_{1}$ and $\phi_{2}$ and apply the phase correction $\phi_{0}$, three Hilbert transforms are required.

In performing the numerical calculations it has been noticed that the axial resolution achievable depends on the choice of $z_{1}$ and $z_{2}$ values which indicates that there are numerical phase errors due to the Hilbert transforms. Therefore, the purpose of this work is to investigate how these phase errors translate to broadening of the ultimate obtainable axial resolution. We have characterized the propagation of errors at both low frequency (small OPD) and high frequency (large OPD) modulation of channeled spectra employed in the calibration process.

\section{EXPERIMENTAL SETUP}

A sketch of the spectrometer based UHR SD-OCT setup is depicted in Fig. 1. Near infrared (IR) light produced by SuperK Extreme from NKT Photonics, Denmark, is guided to a coupler and split into two paths denoted $\mathrm{S}$ and $\mathrm{R}$. Both rays entering the free space part of $\mathrm{S}$ and $\mathrm{R}$ are collimated to a beam width of $3.4 \mathrm{~mm}$. In $\mathrm{S}$ two galvanometer scanners, equipped with mirrors, perform XY raster scanning after which the beam is finally focused with a microscope objective yielding a lateral resolution of $\sim 6 \mu \mathrm{m}$. In $\mathrm{R}$ the beam enters a dispersion compensation (DC) block and is finally reflected by a plane mirror. Reflected light from the two paths mix in the coupler and an interferogram is measured by a spectrometer from Wasatch Photonics, USA.

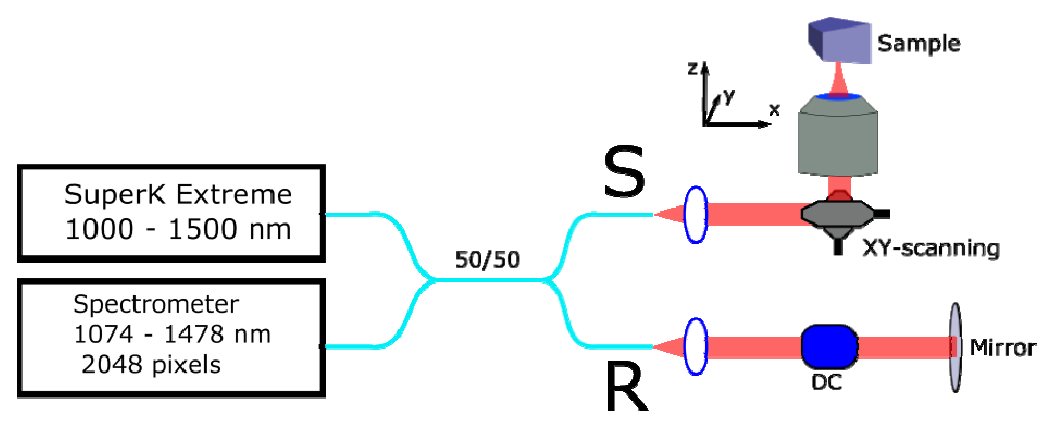

Figure 1: Sketch of experimental setup.

\section{RESULTS}

To investigate the impact of the specific choice of calibration interferograms on resolution, a mirror is employed as a sample in the sample arm S (Fig. 1) and a series of interferograms are recorded translating the mirror in the reference arm R in steps of $100 \mu \mathrm{m}$. Two more spectra, a sample (R-arm blocked) and reference spectra (S-arm blocked), are collected for the purpose of normalization. Several interferogram pairs are chosen for calibration and DC after which A-scans are calculated. The peak in each A-scan, representing the point spread function for a single layer target, is fitted using a least squares procedure with a Gaussian function employing three free parameters: peak height, its width and its OPD position. An example of A-scans obtained, each fitted with a Gaussian function, is presented in Fig. 2. From each fit, a full width half maximum (FWHM) is extracted that 
defines the achievable axial resolution at each respective OPD value. A comparison of axial resolutions for different choices of calibration interferograms are presented in Fig. 3. The OPD values associated with two calibration interferograms (providing phases corresponding to $\phi_{1}$ and $\phi_{2}$ in eq. (3)) are termed $\mathrm{M}_{1}$ and $\mathrm{M}_{2}$. It is apparent that the resolution is more sensitive to the choice of M1 than to the choice of $\mathrm{M}_{2}$ (assuming $\mathrm{M}_{1}<\mathrm{M}_{2}$ ). Furthermore, the choice of $\mathrm{M}_{1}$ becomes extremely critical when approaching half of the axial imaging range, as most pronounced for $\mathrm{M}_{1}=750 \mu \mathrm{m}$ and $\mathrm{M}_{2}=1150 \mu \mathrm{m}$.

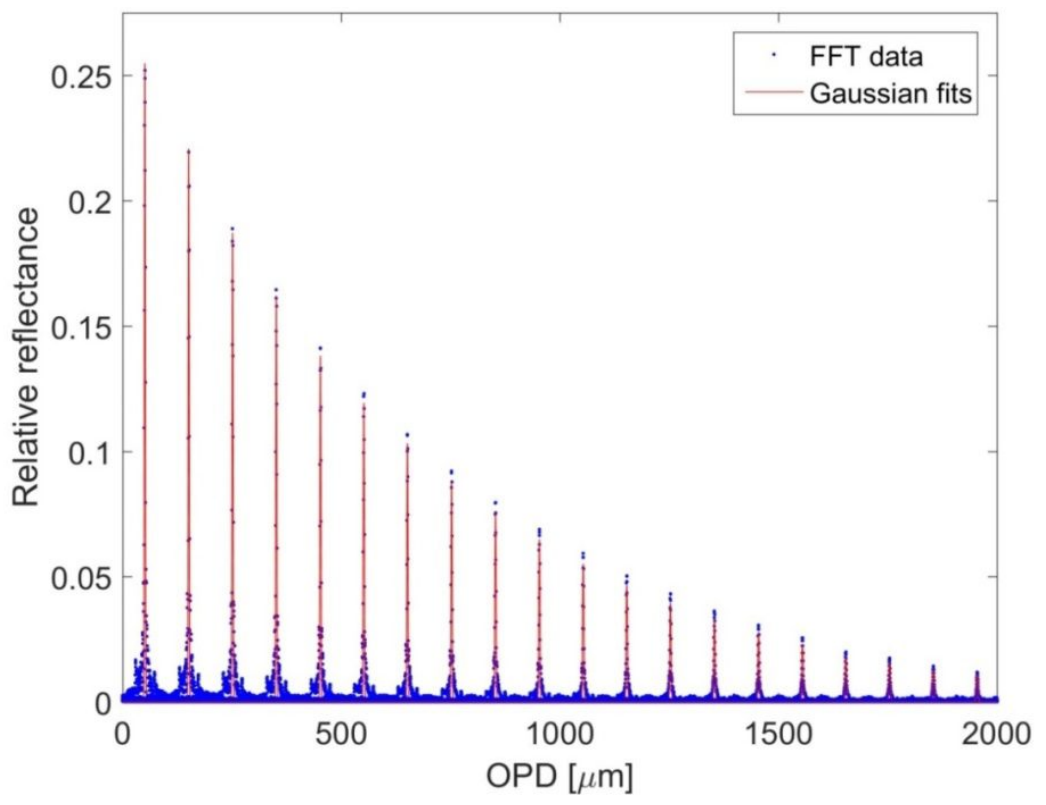

Figure 2: A-scans produced from experimental interferograms at $\mathrm{OPD}=150 \mu \mathrm{m}$ andOPD $=150 \mu \mathrm{m}$ for a sample mirror moved in steps of $100 \mu \mathrm{m}$ and associated Gaussian fits. 


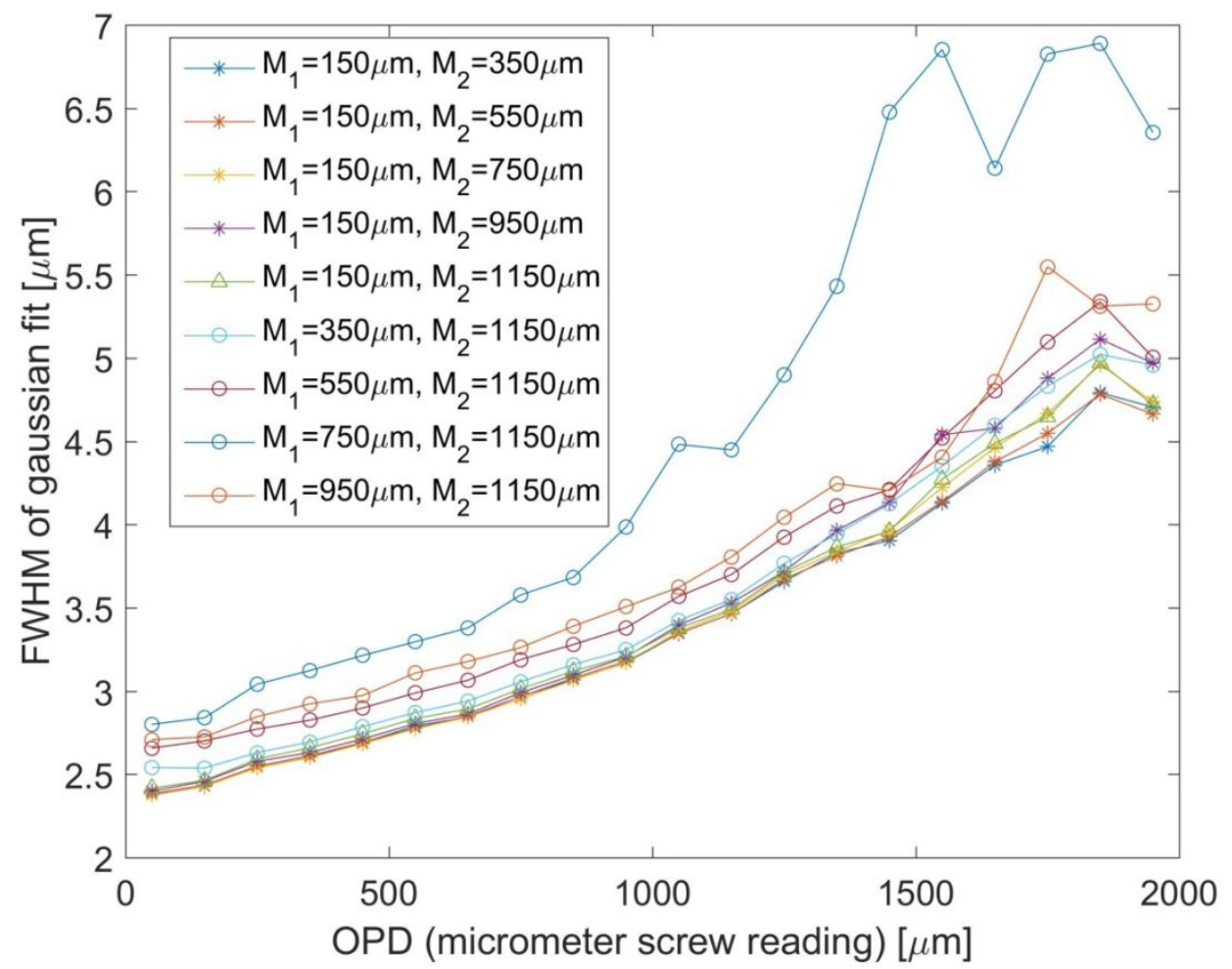

Figure 3: Axial resolution as function of OPD for a number of calibration interferogram pairs denoted by OPDs $\left(\mathrm{M}_{1}\right.$ and $\mathrm{M}_{2}$ ) ranging from $150 \mu \mathrm{m}$ to $1150 \mu \mathrm{m}$. The evaluation is performed in two series where either $\mathrm{M}_{1}$ is fixed and varying $\mathrm{M}_{2}$ or vice versa, as shown in the legend.

To investigate solely how the choice of OPDs of the calibration interferograms (and hence the oscillation period in $\mathrm{k}$-space) influences the resolution emanating from the numerical uncertainties generated in applying the Hilbert transform, we have repeated the calculation of A-scans. To study this phenomenon, simulated harmonic interferograms defined as $\cos \phi_{d}$ with $\phi_{d}(i)=2 z k, k=2 \pi / \lambda_{0}$ are processed. In this way we test the numerical errors generated in the signal processing only associated with the choice of OPDs which translates to variations in the final resolution. 


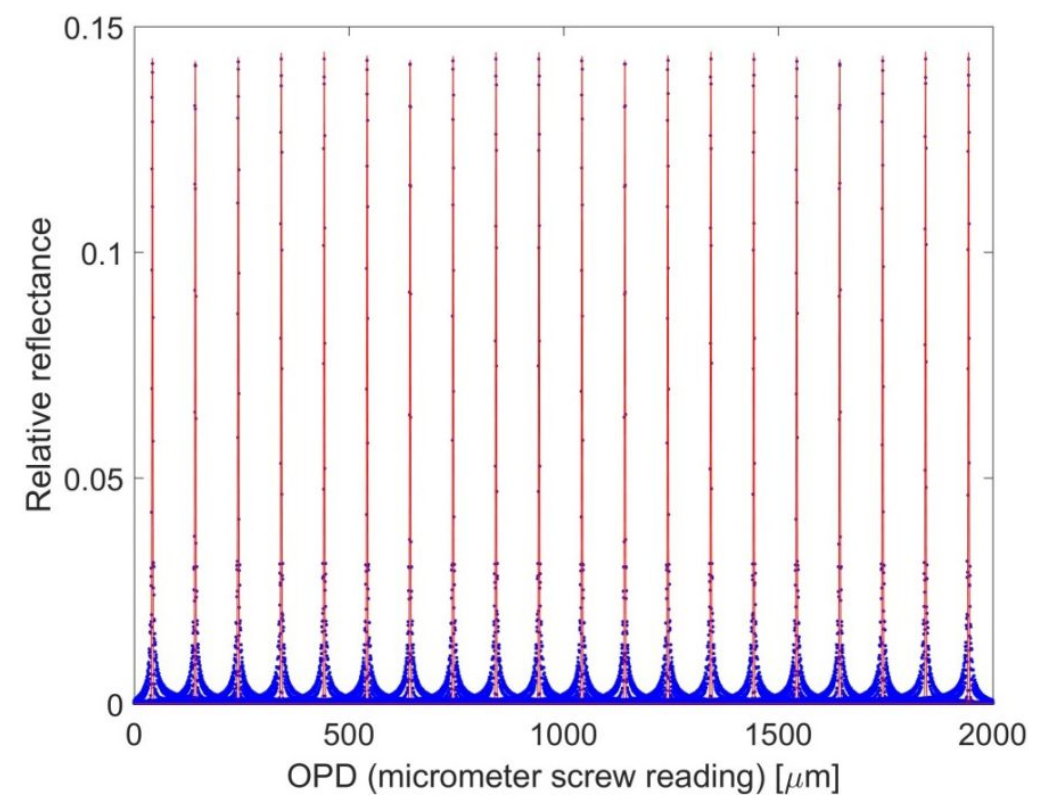

Figure 4: A-scans produced from simulated interferograms at $\mathrm{OPD}=350 \mu \mathrm{m}$ and $\mathrm{OPD}=1150 \mu \mathrm{m}$ with associated fits similar to the ones in Fig. 2.

Fig. 4. These show no decay with OPD.

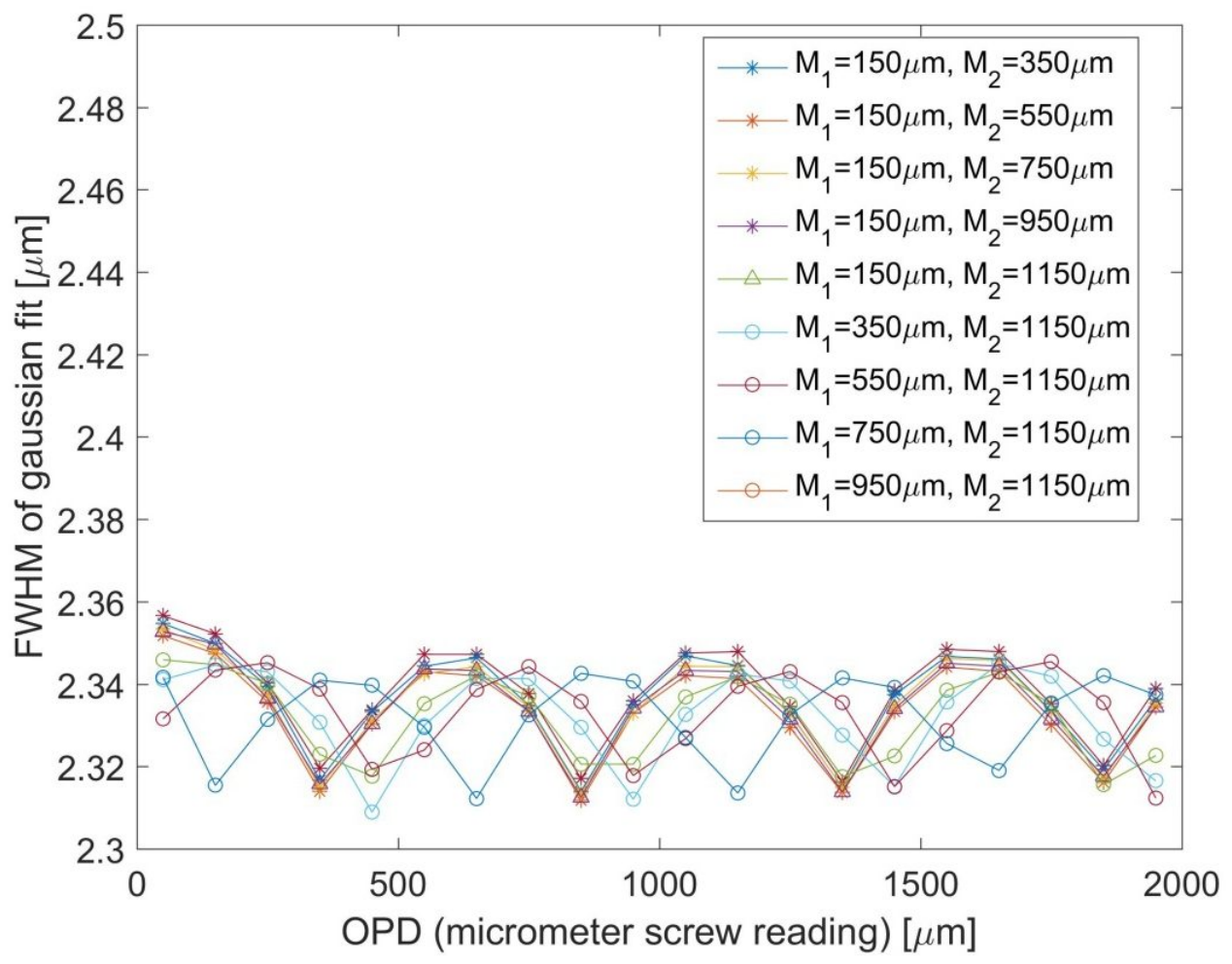

Figure 5: Axial resolution as function of OPD is plotted for calibration interferogram pairs identical to the ones presented in Fig. 3. denoted by OPDs $\left(\mathrm{M}_{1}\right.$ and $\left.\mathrm{M}_{2}\right)$ ranging from $150 \mu \mathrm{m}$ to $1150 \mu \mathrm{m}$. 
Axial resolutions for identical choices of calibration interferograms to those used Fig. 3, are calculated and represented Fig. 5. Only very small changes in the axial resolution are noticed for the span of calibration interferogram choices, that are of the order of $10 \mathrm{~nm}$. We believe that these variations are due to the slight changes in the sampling of the point spread functions.

In conclusion we tested the complete processing module utilised by conventional spectral domain processing in obtaining A-scans from interferograms. The processing module involves three Hilbert transforms where each introduces its own finite phase error. We tested the significance of these numerical errors for both experimentally obtained data and simulated data, the latter constituting ideal harmonic signals. We see that the numerical errors occurring from the processing module are insignificant for all combinations of calibration interferograms when inputting ideal harmonic interferograms. A resolution sensitivity dependence on the choice of calibration interferograms is noticed for the experimentally obtained data. This demands further investigations to identify the exact cause of such a dependence.

A possible cause is illustrated by the trend of curves in Fig. 3. They show that the larger the OPD, the worse the axial resolution. In opposition, Fig. 5 show oscillations of the axial resolution with OPD around a constant value. This comparison suggests that experimentally, the larger the OPD, the smaller the bandwidth of the spectra are modulated by interference as seen by the Hilbert transform. This is not the case with the idealised periodic functions used in Fig. 5, where their effective bandwidth (bandwidth recognised by the Hilbert transform) is maintained constant. It may be that those choices of pairs $\mathrm{M}_{1}$ and $\mathrm{M}_{2}$ may coincide with variations in the effective bandwidth of the spectral modulation, less than the spectrum of the signal incident on the OCT interferometer.

\section{REFERENCES}

[1] Huang, D. et al. "Optical coherence tomography". Science 254, 1178 (1991).

[2] Drexler, W. \& Fujimoto, J. (eds.) "Optical Coherence Tomography”, Springer International Publishing, Switzerland, (2015).

[3] Makati, S. et al. "Full-range, high-speed, high-resolution 1- $\mu \mathrm{m}$ spectral-domain optical coherence tomography using BM-scan for volumetric imaging of the human posterior eye", Optics Express 16. 12 $8406-8420(2008)$

\section{ACKNOWLEDGEMENTS}

N. M. Israelsen, O. Bang and A. Podoleanu acknowledge support from Innovation Fund Denmark through the Shape OCT grant No. 4107-00011A. 